\title{
Thermal properties of metal matrix composites with planar distribution of carbon fibres.
}

\author{
Valerio Oddone ${ }^{*, 1}$ and Stephanie Reich, \\ ${ }^{1}$ Department of Physics, Free University Berlin, Arnimallee 14, 14195 Berlin, Germany
}

Keywords Thermal conductivity, thermal expansion, metal matrix composite, carbon fibres.

* Corresponding author: e-mail valerio.oddone@gmail.com, Phone: +49 83854294

\begin{abstract}
High thermal conductivity and a tunable coefficient of thermal expansion are essential properties for heat management materials operating in a wide temperature range. We combine both properties in a composite with a low density metal matrix reinforced with pitch-based carbon fibres. The thermal conductivity of the metal matrix was increased by $50 \%$, the thermal expansion coefficient was reduced by a factor of five. The samples were produced by powder metallurgy and have a planar random distribution of fibres, leading to high performance in two dimensions.
\end{abstract}

1 Introduction The performance of electronic components grows together with the power density, making the heat dissipation challenging. The high thermal conductivity (TC) of heat sinks cannot be exploited to the maximum if the interface to the cooled electronic component is poor due to a thermal paste layer. Direct, solid-solid interfaces achieve a lower thermal resistance, however require matching coefficients of thermal expansion (CTE) [1-3].

Several research works combined the high performance of carbon based materials (graphite, graphene, carbon nanotubes, carbon fibres) as fillers for metal matrix composites to meet the described $\mathrm{TC}$ and $\mathrm{CTE}$ requirements [4-10]. Fillers with low surface/volume ratio (i.e. large particles rather than nanomaterials) minimise the deleterious interface thermal resistance [11] leading to higher TC.

In this paper, we analyse the performance of pitchbased carbon fibres (CF) as filler for thermal management materials. Pitch-based $\mathrm{CF}$ have a TC as high as $1000 \mathrm{~W} / \mathrm{mK}$ [12], two orders of magnitude higher than the typical polyacrylonitrile-based CF used in engineering. Their suitability as a filler for metal matrix composites was shown in several studies and applications [13], however, in most cases with unidirectional distribution of carbon fibres, leading to high TC and low CTE along one axis [8,14-17]. Most previous studies treated the performance of Solvay P100S fibres [6,15,17], whose production was stopped a decade ago.

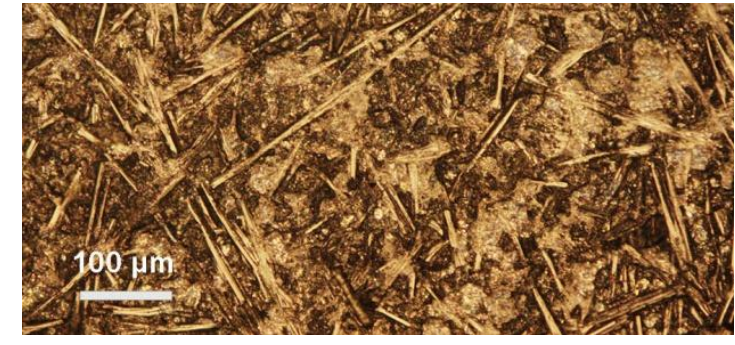

Surface of a metal matrix composite with $50 \%$ carbon fibre strengthening (optical microscope, 20x magnification).

Due to the typical flat geometry of electronic components, the low CTE of the heat sinks is required in at least two dimensions, which can be provided by a planar distribution of the carbon fibres $[4,18]$. We obtain this distribution by powder metallurgy with a uniaxial compression of the matrix-fibres mixture, improving the TC and drastically reducing the CTE in the plane in comparison to the metal matrix. The high planar TC is well suited for thin devices in which cooling fan and electronic component cannot be stacked and are laterally connected by heat pipes. We also produced materials with a gradient in the CF concentration for the use as heat sinks. They may be used to match the CTE of the electronic component on one face and the CTE of copper heat pipes on the other face, distributing thermal stress over a large volume.

Here we present composites with a density below aluminium and higher specific TC than copper, making them ideal for portable and aerospace applications. Thank to the mechanical properties and the high aspect ratio, the carbon fibres build a stable mesh in the metal matrix (see picture in the abstract), leading to excellent machinability and allowing up to $80 \mathrm{vol} \%$ concentration of the filler - a clear advantage in comparison to graphite and nanomaterials.

\section{Experimental}

2.1 Sample preparation Highly thermally conductive $(900 \mathrm{~W} / \mathrm{mK})$ pitch-based carbon fibres XN100-25M (milled fibres, 250x10 $\mu \mathrm{m}$ ) and XN100-03Z (chopped fi- 
bres, 3000x10 $\mu \mathrm{m})$ were provided by the Nippon Graphite Fiber Corporation. Al2024 alloy powder by Ecka Granules Germany, Mg-0.9Ca alloy powder by the HelmoltzZentrum Geesthacht. The metal powders were mixed in a mortar with up to $65 \mathrm{vol} \%$ of chopped fibres or up to $80 \mathrm{vol} \%$ of milled fibres. The powder mixtures were sintered by Spark Plasma Sintering (Dr. Sinter 211-Lx) with following paramenters:

- Temperature: $550^{\circ} \mathrm{C}(\mathrm{Al} 2024), 600^{\circ} \mathrm{C}(\mathrm{Mg}-0.9 \mathrm{Ca})$

- Heating rate: $50 \mathrm{~K} / \mathrm{min}$ (25 mm samples), $100 \mathrm{~K} / \mathrm{min}$ (6 mm samples)

- Pressure: 44-50 MPa, uniaxial

- Atmosphere: vacuum of $1 \mathrm{~Pa}$.

The uniaxial pressing aligns the $\mathrm{CF}$ in a plane (see $\mathrm{Sec}$ tion 3.1). We define the directions $\mathrm{x}, \mathrm{y}$ parallel and $\mathrm{z}$ perpendicular to the plane. Powder mixtures with variable $\mathrm{CF}$ content can be layered in the crucible in order to obtain compositionally graded materials distributing thermal stresses in a large volume (Figure 1).

Magnesium-based samples were annealed for $2 \mathrm{~h}$ at $350^{\circ} \mathrm{C}$, aluminium-based samples were $\mathrm{T} 6$ treated $(2 \mathrm{~h}$ at $490^{\circ} \mathrm{C}$, water quenching, $12 \mathrm{~h}$ at $190^{\circ} \mathrm{C}$ ).

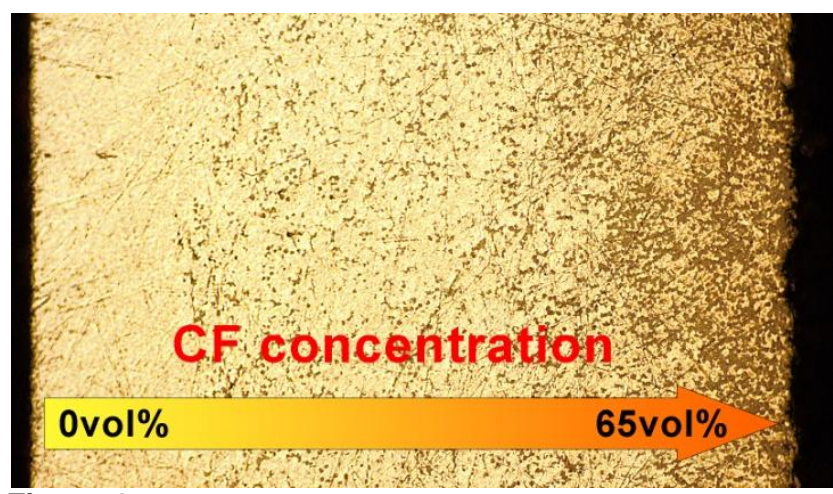

Figure 1 Optical microscope picture of a compositionally graded sample, cut section of $1.5 \mathrm{~mm}$ thickness. Al2024 matrix and 0 to 65 vol\% CF.

2.2 Thermal characterisation Thermal diffusivity was measured by the flash method with a Netzsch LFA447 NanoFlash for disc-shaped samples with $25 \mathrm{~mm}$ diameter and 1-2 mm thickness. In plane measurements were performed by the radial heat flow method [19]. The thermal conductivity was obtained by multiplication with the density and the specific heat capacity, determined respectively by the geometric method and the rule of mixture. The CTE was measured by dilatometry with a Linseis L75XH1OOO in four cycles between $50^{\circ} \mathrm{C}$ and $150^{\circ} \mathrm{C}$ with heating rate of $0.5 \mathrm{~K} / \mathrm{min}$. Cylindrical samples with $6 \mathrm{~mm}$ diameter and 4 $6 \mathrm{~mm}$ thickness were measured. Details about the thermal characterisation are described in our previous work [20]

2.3 Mechanical characterisation Tensile strength in $\mathrm{x}, \mathrm{y}$-direction was measured with a Zwick Z010 tensile tester. The samples used for thermal characterization (25 mm diameter, 1-2 mm thickness) were milled to a dog- bone shape with a gage length of $8 \mathrm{~mm}$ and width of $5 \mathrm{~mm}[20]$.

3 Results and discussion The addition of up to $65 \mathrm{vol} \%$ of carbon fibres enhanced the TC and reduced the CTE in the $\mathrm{x}, \mathrm{y}$-plane. The density of samples with aluminium matrix decreased to $2200-2400 \mathrm{~kg} / \mathrm{m}^{3}$, whereas samples with magnesium matrix it increased to 1800 $2000 \mathrm{~kg} / \mathrm{m}^{3}$. The densification (density measured by geometric method divided by the theoretical density) ranged from $97-100 \%$ for pure $\mathrm{Al} 2024,96-99 \%$ for pure $\mathrm{Mg}$ $0.9 \mathrm{Ca}, 94-97 \%$ for $65 \mathrm{vol} \%$ concentration of $\mathrm{CF}$ in both metals. The TC and CTE results with milled (long) and chopped (short) fibres were similar for identical matrix-tofibre ratio, but the samples with milled fibres were easier to sinter, had up to $30 \%$ lower porosity and higher mechanical stability, therefore only the results for the latter are presented. Since the composites with chopped fibres had similar TC in spite of the higher porosity, significantly higher TC shall be expected if a more efficient densification is achieved. In order to reach the optimal properties, we chose the highest possible temperature in the semiliquid state (at higher temperature the viscosity of the matrix is too low, resulting in separation of the filler by buoyancy and liquid metal flowing out of the crucible). The high temperature helped overcoming the strong oxide layer of aluminium and magnesium and reduced the porosity. We did not observe any major effect changing the heating rate, sintering time and pressure. Samples with up to $80 \mathrm{vol} \%$ of CF could be sintered. However their densification was below $80 \%$, resulting in low TC and mechanical weakness.

3.1 Orientation of the fibres Spark plasma sintering was shown to lead to a high degree of alignment in the case of graphite platelets [5]. We expect a comparable effect for the carbon fibres in the metal matrix. For a quantitative evaluation of the orientation, we measured the compression ratio $\varepsilon$ of the sinter process (defined as ratio of the density of the powder mixture to the density of the sintered material). This grows linearly from $\varepsilon=1.8$ at $0 \mathrm{vol} \%$ of fibres to $\varepsilon=4.5$ at $65 \mathrm{vol} \%$. The fibres have an isotropic distribution in the powder mixture. Considering the movement of stiff fibres with fixed length within the compressing matrix, the reorientation of a fibre with angle $\theta^{*}$ to the $\mathrm{z}$-axis is given by

$$
\theta=\operatorname{arccot} \frac{\cot \theta^{\prime}}{\varepsilon} .
$$

Differentiating the inverse of this equation leads to the orientation distribution function $\phi(\theta)$.

$$
\phi(\theta)=\frac{\varepsilon}{\varepsilon^{2} \cos ^{2} \theta+\sin ^{2} \theta} .
$$

This function is used for the calculation of the average orientation $\left\langle\cos ^{2} \theta\right\rangle$ of the fibres according to Nan et al. [21]. We find values from 0.23 for minimal concentration of fibres to 0.12 for $65 \mathrm{vol} \%$ of fibres. The probability 
density that the orientation of a fibre is between $\theta$ and $\theta+$ $d \theta$ is given by $\phi(\theta) \cdot \sin (\theta)$.

3.2 Thermal conductivity For both metals the TC in the $\mathrm{x}, \mathrm{y}$-plane increases and the $\mathrm{TC}$ along $\mathrm{z}$ decreases for higher CF content (see Fig. 2), confirming the fibre alignment through the anisotropic parameters. The specific thermal conductivity (TC/density) is for both metal matrices at 50-65vol\% CF concentration approximately twice as high as in pure copper. For a high thermal conductivity, the highest possible sintering temperature is essential. For a $\mathrm{CF}$ concentration above $50 \mathrm{vol} \%$, a $50^{\circ} \mathrm{C}$ lower sintering temperature lead to $20-30 \%$ lower TC. We did not observe any significant influence of the sintering pressure and of the heating rate.

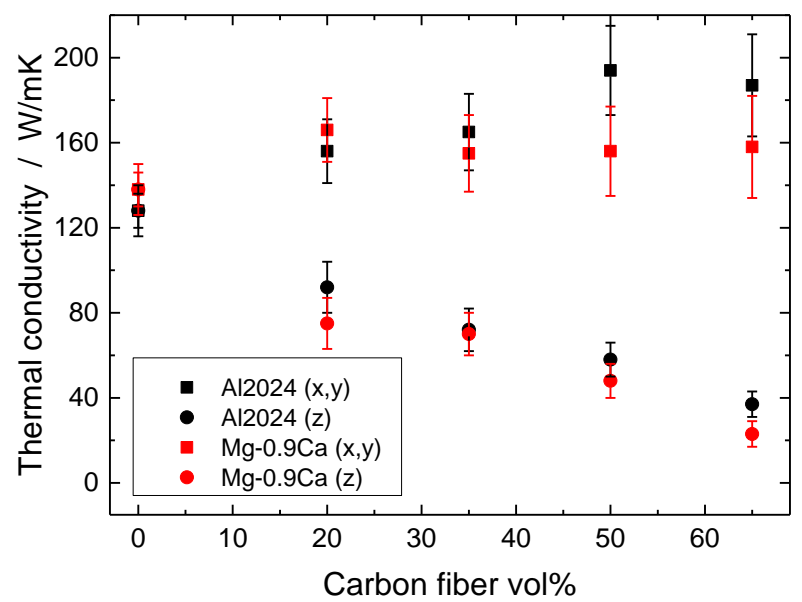

Figure $2 \mathrm{TC}$ of $\mathrm{Al} 2024$ and $\mathrm{Mg}-0.9 \mathrm{Ca}$ composites for variable $\mathrm{CF}$ content (Notice that the $\mathrm{x}, \mathrm{y}$ and $\mathrm{z}$ value at $0 \mathrm{vol} \%$ concentration are overlapping).

To estimate a predicted TC of the composite, we assume that the fibres have zero TC in radial direction and according to the manufacturer $\lambda_{f}=900 \mathrm{~W} / \mathrm{mK}$ in axial direction (the TC in radial direction is assumed to be similar to graphite out-of-plane due to the inner structure [22], i.e. $5-10 \mathrm{~W} / \mathrm{mK}$ and reduced due to the interface resistance [23], therefore negligible). For the matrix we use the measured TC without fibres $\lambda_{m}=130-140 \mathrm{~W} / \mathrm{mK}$ and consider it as isotropic. The thermal interface resistance for our fibre length of $250 \mu \mathrm{m}$ is negligible [23]. We model our composite with the laminate plate theory from Villière et al. [24], i.e. as a stack of x,y-aligned plates. The fibres in one plate have unidirectional orientation in $\mathrm{x}$ or $\mathrm{y}$ direction and are slightly tilted to have the average orientation $\langle\theta>$ to the z-axis (Section 3.1). The TC parallel to the fibre direction is given [23]

$$
\lambda^{\|}=(1-\Phi) \cdot \lambda_{m}+\Phi \cdot \lambda_{f},
$$

where $\Phi$ is the volume fraction of the fibres. Perpendicular to the fibres we obtain

$$
\lambda_{\perp}=\lambda_{m} \frac{1-\Phi}{1+\Phi}
$$

(Note that the fibres reduce the thermally conducting cross section and in addition increase the length of the conducting path).

By trigonometry, the $\mathrm{TC}$ of one plate in $\mathrm{x}, \mathrm{y}$ and $\mathrm{z}$ direction is found. For the TC in $\mathrm{x}, \mathrm{y}$ direction (parallel to the plate), a model of parallel thermal resistances is applied. For the $\mathrm{TC}$ in $\mathrm{z}$ direction (perpendicular to the plate) a model of serial thermal resistances is applied.

Comparing the measured and calculated TC we find that the measured TC of the composite at $65 \mathrm{vol} \%$ fibre concentration is $40 \%$ lower in $\mathrm{x}, \mathrm{y}$-direction and $60-70 \%$ lower in z-direction than predicted. Assuming the manufacturer data is correct, we suggest two possible factors leading to the lower experimental values:

- At the ends of the fibres the CTE-mismatch produces tiny air bubbles, strongly deteriorating the thermal interface [25]

- Due to the uniaxial compression, the pores have oblate shape in the $\mathrm{x}, \mathrm{y}$ plane, creating additional anisotropy in the matrix

In this case, a more efficient densification has a great potential to further enhance the $\mathrm{TC}$ of the composite.

It was also suggested that the filler has a deleterious influence on the microstructure of the matrix at the interface [26]. However, this effect is probably minor for our macroscopic fibres in comparison to composites with nanosized fillers.

3.3 Thermal expansion Similarly to the results for the TC, we observed for growing CF concentration a strongly decreasing CTE in the x,y-plane (Fig. 3). Pitch based carbon fibres have a CTE of $-1.6 \mathrm{ppm} / \mathrm{K}$ [27]. The elasticity modulus of the fibres is with $>800 \mathrm{GPa}$ (manufacturer data) significantly higher than the modulus of the matrix $(73 \mathrm{GPa}$ for AL2024 and $45 \mathrm{GPa}$ for magnesium [28]). Hence, the $\mathrm{x}, \mathrm{y}$-CTE of composites with high filler concentration should approach the CTE of the fibres. This is in accordance with our experimental results and proves the good adhesion between matrix and filler. The CTE in z-direction reaches a maximum at $35 \mathrm{vol} \%$ of fibres and decreases for higher concentration. The initial CTE increase can be clearly explained with the Poisson effect of the matrix (Poisson ratio of 0.35 for both metals [28]): the expansion in $\mathrm{x}, \mathrm{y}$-direction is restricted by the fibres, which corresponds to a compressive strain of the matrix in $\mathrm{x}, \mathrm{y}$-direction, followed by an expansion in $\mathrm{z}$ direction. For higher filler concentrations (50-65vol\%), this effect is inverted since the low transverse CTE of the fibre phase ( $8.8 \mathrm{ppm} / \mathrm{K}$ [27]) dominates, leading to a drop in the overall CTE in z-direction. Moreover, the Poisson effect in the fibres (the fibres are axially overstretched and shrink radially) causes a further, slight reduction of their transverse CTE. 


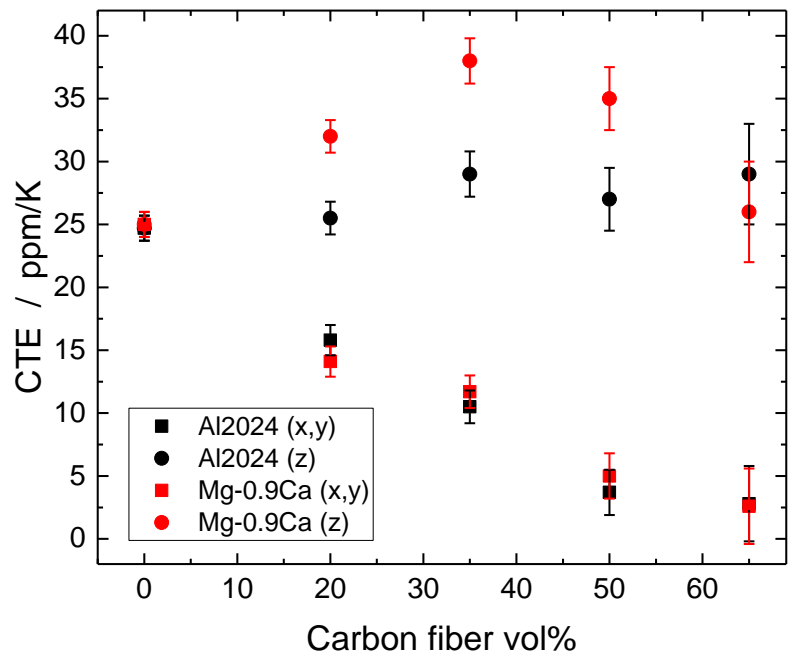

Figure $3 \mathrm{CTE}$ of $\mathrm{Al} 2024$ and $\mathrm{Mg}-0.9 \mathrm{Ca}$ composites for variable $\mathrm{CF}$ content at $100^{\circ} \mathrm{C}$ (Notice that the $\mathrm{x}, \mathrm{y}$ and $\mathrm{z}$ value at $0 \mathrm{vol} \%$ concentration are overlapping).

In similar composites with graphite flakes instead of $\mathrm{CF}$ as filler we observed the opposite effect, i.e. a small reduction of the $\mathrm{x}, \mathrm{y}-\mathrm{CTE}$, but a major reduction of the $\mathrm{z}$ CTE down to negative values $[20,29]$. CF-metal composites and graphite-metal composites have thus complementary properties, covering a wide range of applications.

3.4 Tensile strength For completeness, the tensile strength of the produced materials was determined. As shown in Fig. 4, the strength exponentially decreases for higher CF concentration, however is higher than metalgraphite composites with comparable filler concentration [20]. Although the used CF have a tensile strength of $>3 \mathrm{GPa}$ (manufacturer data), the fibres introduce weak points in the matrix causing an overall reduced strength. The samples remain stable and can be easily machined at up to $65 \mathrm{vol} \% \mathrm{CF}$ concentration.

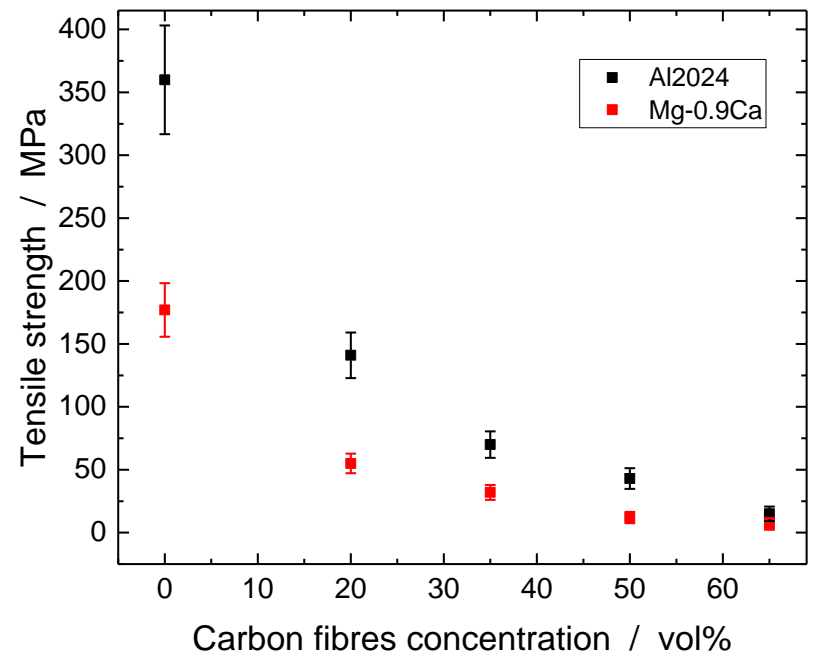

Figure 4 Tensile strength of Al2024 and Mg-0.9Ca composites for variable $\mathrm{CF}$ content.

4 Summary In conclusion, we sintered metal matrix composite reinforced with pitch-based carbon fibres. In the orientation plane of the fibres the measured thermal conductivity approaches $200 \mathrm{~W} / \mathrm{mK}$ while the coefficient of thermal expansion can be tuned down to $3 \mathrm{ppm} / \mathrm{K}$. In particular, by variation of the carbon fibres concentration within a component, different CTE values at the two sides can be achieved. The density of the composites (1800$2400 \mathrm{~kg} / \mathrm{m}^{3}$ ) is four to five times lower than that of copper. These properties make the sintered materials ideal for heat sinks, in particular for mobile or aerospace applications, where a lightweight construction is essential and high thermal fluctuation represent a big stress for electronic components.

Acknowledgements $\mathrm{VO}$ acknowledges the Evonik Foundation for the financial support. We acknowledge M. Wolff from the Helmoltz-Zentrum Geesthacht for providing the $\mathrm{Mg}-0.9 \mathrm{Ca}$ powders. We thank P. Leibner from the Max Planck Institute of Colloids and Interfaces for use of their equipment for tensile tests.

\section{References}

[1] R. O. Carlson, H. H. Glascock, H. F. Webster, and C. A. Neugebauer, in MRS Proc. (Cambridge Univ Press, 1984), p. 177.

[2] D. D. L. Chung, Materials for Electronic Packaging (Butterworth-Heinemann, Woburn (MA), 1995).

[3] B. Attaf (ed.), Advances in Composite Materials Ecodesign and Analysis (InTech, 2011), pp. 271272.

[4] J. A. Cornie, S. Zhang, R. Desberg, and M. Ryals, Met. Matrix Compos. LLC 7 (2003).

[5] A. Boden, B. Boerner, P. Kusch, I. Firkowska, and 
S. Reich, Nano Lett. 14, 3640 (2014).

[6] S. Rawal, JOM J. Miner. Met. Mater. Soc. 53, 14 (2001).

[7] J. K. Chen and I. S. Huang, Compos. Part B Eng. 44, 698 (2013).

[8] G. Korb, J. Koráb, and G. Groboth, Compos. Part A 29, 1563 (1998).

[9] K. Chu, Q. Wu, C. Jia, X. Liang, J. Nie, W. Tian, G. Gai, and H. Guo, Compos. Sci. Technol. 70, 298 (2010).

[10] T. Etter, M. Papakyriacou, P. Schulz, and P. J. Uggowitzer, Carbon 41, 1017 (2003).

[11] K. Jagannadham, J. Appl. Phys. 110, 74901 (2011).

[12] M. L. Minus and S. Kumar, J. Miner. Met. Mater. Soc. 57, 52 (2005).

[13] W. Buchgraber, G. Korb, T. Schubert, and B. Kempf, in Microstruct. Investig. Anal. Vol. 4 (Wiley Online Library, n.d.), pp. 150-155.

[14] J. Koráb, P. Štefánik, S. `Kavecký, P. Šebo, and G. Korb, Compos. - Part A 33, 577 (2002).

[15] M. Russell-Stevens, R. I. Todd, and M. Papakyriacou, J. Mater. Sci. 41, 6228 (2006).

[16] P. Sebo and P. Stefanik, Int. J. Mater. Prod. Technol. 18, 141 (2003).

[17] D. L. Ellis and D. L. McDanels, Metall. Trans. A 24, 43 (1993).

[18] J.-F. Silvain, A. Veillère, and Y. Lu, J. Phys. Conf. Ser. 525, 12015 (2014).

[19] A. B. Donaldson and R. E. Taylor, J. Appl. Phys. 46, 4584 (1975).

[20] V. Oddone, B. Boerner, and S. Reich, Sci. Technol. Adv. Mater. 18, 1 (2017).

[21] C.-W. Nan, R. Birringer, D. R. Clarke, and H. Gleiter, J. Appl. Phys. 81, 6692 (1997).

[22] I. Mochida, S. Yoon, N. Takano, F. Fortin, Y. Korai, and K. Y. O. K. Gawa, Carbon N. Y. 34, 941 (1996).

[23] A. J. Schmidt, K. C. Collins, A. J. Minnich, and G. Chen, J. Appl. Phys. 107, 104907 (2010).

[24] M. Villière, D. Lecointe, V. Sobotka, N. Boyard, and D. Delaunay, Compos. Part A Appl. Sci. Manuf. 46, 60 (2013).

[25] K. Gharagozloo-Hubmann, A. Boden, G. J. F. Czempiel, I. Firkowska, and S. Reich, Appl. Phys. Lett. 102, 213103 (2013).

[26] B. Chen, S. Li, H. Imai, L. Jia, J. Umeda, M. Takahashi, and K. Kondoh, J. Alloys Compd. 651, 608 (2015).

[27] J. F. Villeneuve, R. Naslain, R. Fourmeaux, and J. Sevely, Compos. Sci. Technol. 49, 89 (1993).

[28] J. S. Wilson, Sensor Technology Handbook (Elsevier, 2005).

[29] I. Firkowska, A. Boden, B. Boerner, and S. Reich, Nano Lett. 15, 4745 (2015). 